Article

\title{
Proposal of UWB-PPM with Additional Time Shift for Positioning Technique in Nondestructive Environments
}

\author{
Nguyen Thi Huyen ${ }^{1} \mathbb{D}$, Nguyen Le Cuong ${ }^{2}$ and Pham Thanh Hiep ${ }^{1, *} \mathbb{C}$ \\ 1 Faculty of Radio-Electronic Engineering, Le Quy Don Technical University, 236 Hoang Quoc Viet, \\ Ha noi 100000, Vietnam; nguyenhuyenhvktqs@gmail.com \\ 2 Faculty of Electronics and Telecommunications, Electric Power University, 235 Hoang Quoc Viet, \\ Ha noi 100000, Vietnam; cuongnl@epu.edu.vn \\ * Correspondence: phamthanhhiep@gmail.com; Tel.: +84-982-535-203
}

Received: 20 July 2020; Accepted: 27 August 2020; Published: 30 August 2020

\begin{abstract}
The ultra-wide band (UWB) technology has many advantages in positioning and measuring systems; however, powers of UWB signals rapidly reduce while traveling in propagation environments, hence detecting UWB signals are difficult. Various modulation techniques are applied for UWB signals to increase the ability for detecting the reflected signal from transmission mediums, such as pulse amplitude modulation (PAM), pulse position modulation (PPM), and so on. In this paper, we propose an ultra-wide band pulse position modulation technique with optimized additional time shift (UWB-PPM-ATS) to enhance the accuracy in locating buried object in nondestructive environments. Moreover, the Levenberg-Marquardt Fletcher algorithm (LMFA) is applied to determine the medium parameters and buried object location simultaneously. The influences of proposed modulation technique on determining system's parameters, such as a propagation time, distance, and properties of the medium are analyzed. Calculation results indicate that the proposed UWB-PPM-ATS gives higher accuracy than the conventional one such as UWB-OOK and UWB-PPM in both homogeneous and heterogeneous environments. Furthermore, the LMFA with the proposed UWB-PPM-ATS outperforms the LMFA with the traditional modulation method, especially for unknown propagation environment.
\end{abstract}

Keywords: UWB-PPM; UWB-OOK; buried objects; nondestructive environment; Levenberg-Marquardt method

\section{Introduction}

With ultra-wide bandwidth, the ultra-wide band (UWB) signal is considered as an ideal locating technique in a short-range with high spatial resolution. As defined by the Federal Communications Commission (FCC), UWB technology has a center frequency higher than $2.5 \mathrm{GHz}$, or if less than $2.5 \mathrm{GHz}$, there must be a minimum bandwidth ratio of 0.2 [1], or the minimum bandwidth must reach $500 \mathrm{MHz}$ [2]. To avoid affecting other narrow band systems, the rules of FCC allow the effective isotropic radiated power (EIRP) level of UWB devices to be lower than $-41.3 \mathrm{dBm} / \mathrm{MHz}$ in the frequencies range of 3.1 to $10.6 \mathrm{GHz}$ [3], so the UWB devices can work for more extended periods than narrow band systems with the same battery power, and due to the use of very narrow pulses, UWB signals are better able to penetrate in the nondestructive environments. General modulation techniques are used for UWB signals such as pulse amplitude modulations (PAMs), On-Off Keying (OOK), and pulse position modulation (PPM) [4]. One can use time-hopping (TH) in UWB systems to create TH-PPM, TH-BPSK signal types [5], or design a generator circuit which generates the 4-th and 5-th order derivative of Gaussian pulses in TH-QPSK system applied to multipath channels [6]. Each modulation technique has a different application range. The choice of the right modulation configuration not only increases the efficiency of system implementation but also maximizes the 
benefits of ultra-wide bandwidth and reduces the complexity of device hardware. In [7], a simple peak detection based on noncoherent UWB receiver is proposed for low data rate wireless sensor networks (WSN) and Internet of things (IoT) applications. It has improved receiver performance with TH-PPM UWB signal. In [8], to reduce the complexity of the TH-UWB receiver, a channel shortening equalizer design method is proposed based on an eigen filter using a new objective function, whereby the proposed system has dramatically reduced the power of channel impulse response, spectral distortion, multiaccess interference, and noise power. Therefore, different UWB signal modulation types have affected the quality and application of the UWB system.

In those modulation techniques, the PPM technique is one of the widely used configurations in UWB systems. Studies on UWB-PPM in wireless communication networks mainly focus on solutions to reduce the conflicts in multiuser access systems; for example, [9] proposed an M-ary PPM modulation configuration for the UWB (M-PPM) system and indicated that the proposed system significantly improved performance compared to systems using direct spreading sequences in the environments with a low signal to noise (SNR) ratio. In [10], Vinod Venkatesan et al. proposed the application of a direct spreading sequence with the optimized UWB-PPM technique for multiaccess systems. The proposed method reduced the impact of multiaccess interference (MAI) and significantly reduced the floor error compared to the orthogonal signal configuration at a large SNR ratio. Besides, there are several studies on improving the quality of the receiver for UWB-PPM signals [11], determining the optimal integration time for the energy detector of the UWB-PPM system [12], and developing a measurement matrix combined with randomly Fourier transform converters for UWB-PPM signals [13]. The combination of PPM symbols and time of arrival (TOA) estimation algorithm using the Sub-Nyquist IR-UWB signal in the IR-UWB device is discussed in [14]. Turbo codes for PPM-IR UWB signals to improve the power spectral density (PSD) power signal density [15] and randomizing the pulses to improve the UWB system [16] were proposed. The noncoherent modulation techniques based on the use of the receiver adaptive thresholds applied to enhanced PPM in the IR-UWB and the direct chaotic communication UWB (DCC-UWB) systems were proposed to improve the bit error rate (BER) performance of the system in a multipath transmission environment [17].

For testing purposes, material penetrating systems using UWB technology to examine nondestructive environments are discussed in [18]; the result indicated that this system can detect imperfect structures of metal. Besides that, the estimation of the layer's thickness based on the processing of the GPR's data with the optimized techniques (such as neural networks) is discussed in $[19,20]$. When using UWB technology in the testing, positioning, or another application in communication to improve the resolution of the systems, one of the main problems is choosing the appropriate modulation technique combined with the receiver's signal processing methods. The selection of a modulation scheme based on determination distance was discussed in [21]. From those results, we can recognize that the correct detection of UWB pulse signals is one of the essential factors which affect the accuracy of the distance estimation technique. In [22], an UWB indoor positioning system is presented to exploit two-way flight time to calculate range measurements to determine the transceiver location based on Pozyz inner algorithm with a range accuracy of $320 \pm 30 \mathrm{~mm}$. In [23], to locate underground personnel in coal mines, an UWB wireless sensor network and time difference of arrival (TDOA) algorithm was proposed, and this system can achieve high-precision positioning in real-time. Furthermore, in the nondestructive environment, direct sequence ultra-wide band (DS-UWB) transmission system with an adaptive pseudo random sequence length is proposed in [24] to reduce processing time and increase positioning accuracy. As mentioned above, the PPM modulation is widely applied to the UWB system, especially for detecting the location of objects; however, the accuracy of estimated results is still low. In this paper, we focus on proposing a new modulation scheme based on PPM modulation to improve the resolution of the estimated distance. The main contributions of this research are listed as follows.

- Firstly, the UWB-PPM signal which is used in determining the distance under the homogeneous and heterogeneous propagation conditions is mathematically analyzed. 
- Secondly, based on the analysis of received signal, the delay time and then the propagation distances are estimated.

- Thirdly, to increase the accuracy of the estimated distance, an enhanced UWB-PPM modulation technique, called ultra-wide band pulse position modulation with additional time shift (UWB-PPM-ATS) is proposed and compared with other techniques such as conventional UWB-PPM, UWB- OOK with the properties of transmission environment is known.

- Finally, the Levenberg-Marquardt nonlinear estimation algorithm is applied to estimate the system parameter and the target when the propagation environment is unknown.

The remainder of this paper is organized as follows. Section 2 describes the system model for estimating distances in nondestructive environments. The proposed system model and the parameters are presented in Section 3. The simulation results are provided in Section 4, and finally, conclusions and further work are discussed in Section 5.

\section{System Model}

A distance measurement and positioning system using UWB technology is illustrated in Figure 1, where $d_{i}$ and $\varepsilon_{i}$ are the thickness and the relative permittivity of the $i^{\text {th }}$ layer in the nondestructive environment, respectively. $s(t)$ denotes the transmitted pulse signals, and $r(t)$ is the received signal.

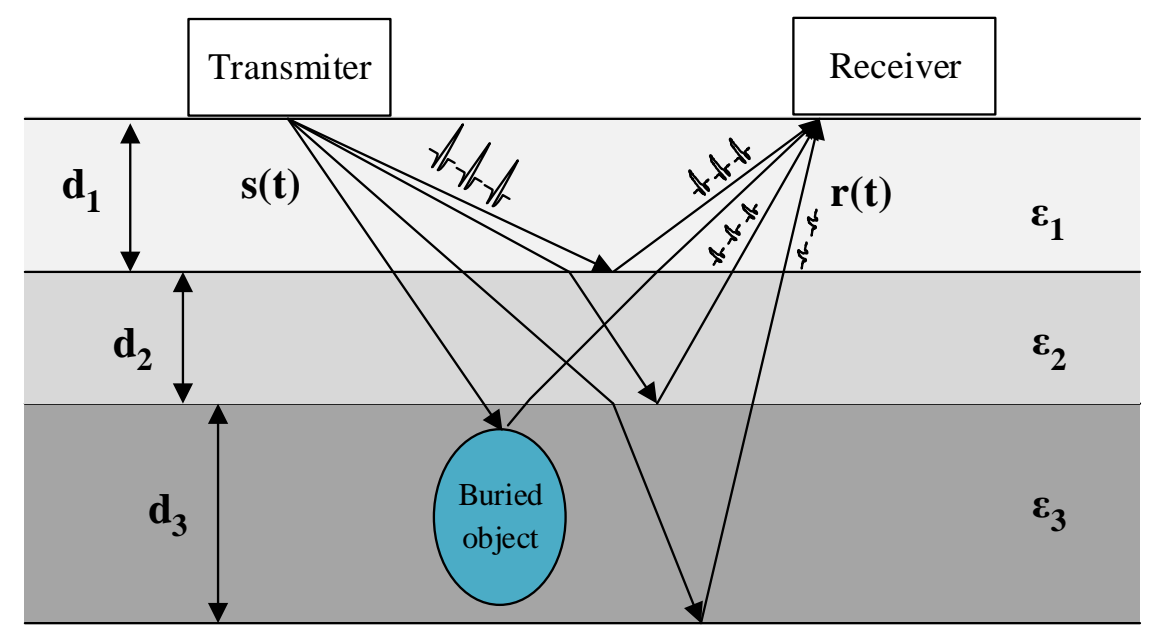

Figure 1. The distance measurement and positioning system in the nondestructive environments.

For the environment with three layers and one buried object, the $r(t)$ is represented by

$$
\begin{aligned}
r(t) & =\sum r_{i}(t)+r_{o b}(t)+n(t), \\
r_{1}(t) & =A_{1} s\left(t-\tau_{1}\right), \\
r_{2}(t) & =A_{1} A_{2} s\left(t-\tau_{1}-\tau_{2}\right), \\
r_{3}(t) & =A_{1} A_{2} A_{3} s\left(t-\tau_{1}-\tau_{2}-\tau_{3}\right), \\
r_{o b}(t) & =A_{o b} s\left(t-\tau_{o b}\right),
\end{aligned}
$$

where $A_{i}$ denotes the amplitude factor which represents the reflection and transmission properties of the propagation environment, and its value depends on the properties of the $i$ th layers, $\tau_{i}$ is the traveling time of UWB pulse in the $i$ th layer. $A_{o b}$ and $\tau_{o b}$ are respectively the amplitude and traveling time of the signal which is reflected from the buried object. $n(t)$ represents the additive white Gaussian noise.

At the receiver side, the procedure for determining the position of buried object is indicated in Figure 2. Accordingly, the position of buried object is determined by the distance from it to 
the transceiver. The LMFA method is applied to calculate the relative permittivity of environment and these distances.

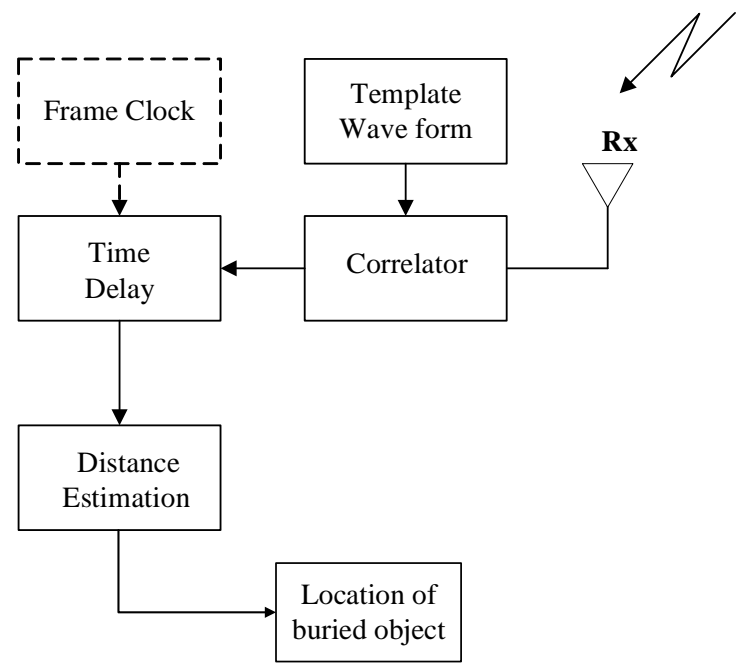

Figure 2. The procedure for determining the position of buried object.

In the UWB system, $s(t)$ is generated based on Gaussian [25] or Hermite [26] functions and their derivatives. Compared to Gaussian pulses, the Hermite pulse is useful for parallel data transmission with high data rates, but it is hard to achieve in the real world [27]. Therefore, the Gaussian pulses UWB signals are applied in this work. A typical Gaussian pulse usually takes the form [25]:

$$
g(t)=A_{p} e^{-2 \pi\left(\frac{t}{\mu_{p}}\right)^{2}},
$$

where $A_{p}$ denotes the amplitude of pulse, $\mu_{p}$ is a factor which influences the amplitude and the width of Gaussian pulse, also called time normalization factor, the width of a pulse becomes narrower when the $\mu_{p}$ is reduced. The $n^{\text {th }}$ derivative of Gaussian pulse, named $n^{\text {th }}$-order monocycle, is

$$
g_{n}(t)=A_{n p} \frac{d^{n}}{d t^{n}} e^{-2 \pi\left(\frac{t}{\mu_{p}}\right)^{2}},
$$

where $A_{n p}$ is the amplitude of the $n^{\text {th }}$-order monocycle. The shapes of different types of Gaussian monocycles are indicated in Figure 3.
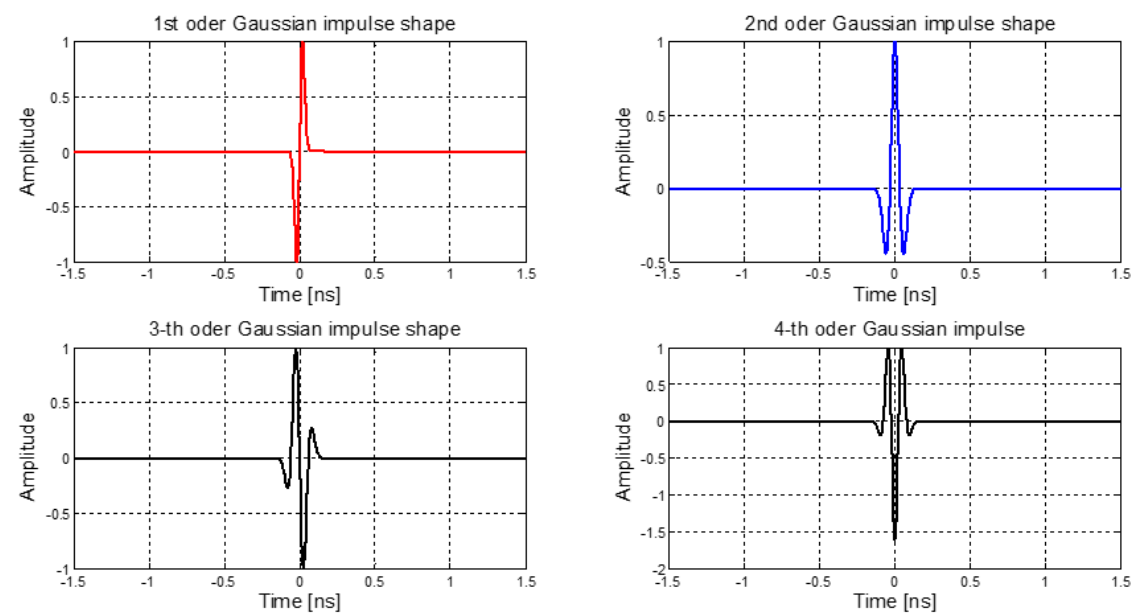

Figure 3. The different types of Gaussian monocycles. 
The selected Gaussian pulse shape must meet FCC requirements about power and frequency range used. According to Figure 4, it is observed that for the power spectral density (PSD), only the first-order Gaussian monocycle does not comply with the FCC requirements about the effective isotropic radiated power (EIRP) level. In this paper, we restrict our performance analysis of UWB system to the fourth-order Gaussian monocycle in (4). However, our method can be applied for any Gaussian pulse shape.

$$
g_{4}(t)=A_{p}\left[-12 \pi+96 \pi^{2}\left(\frac{t}{\mu_{p}}\right)^{2}-64 \pi^{3}\left(\frac{t}{\mu_{p}}\right)^{4}\right] e^{-2 \pi \cdot\left(\frac{t}{\mu_{p}}\right)^{2}} .
$$

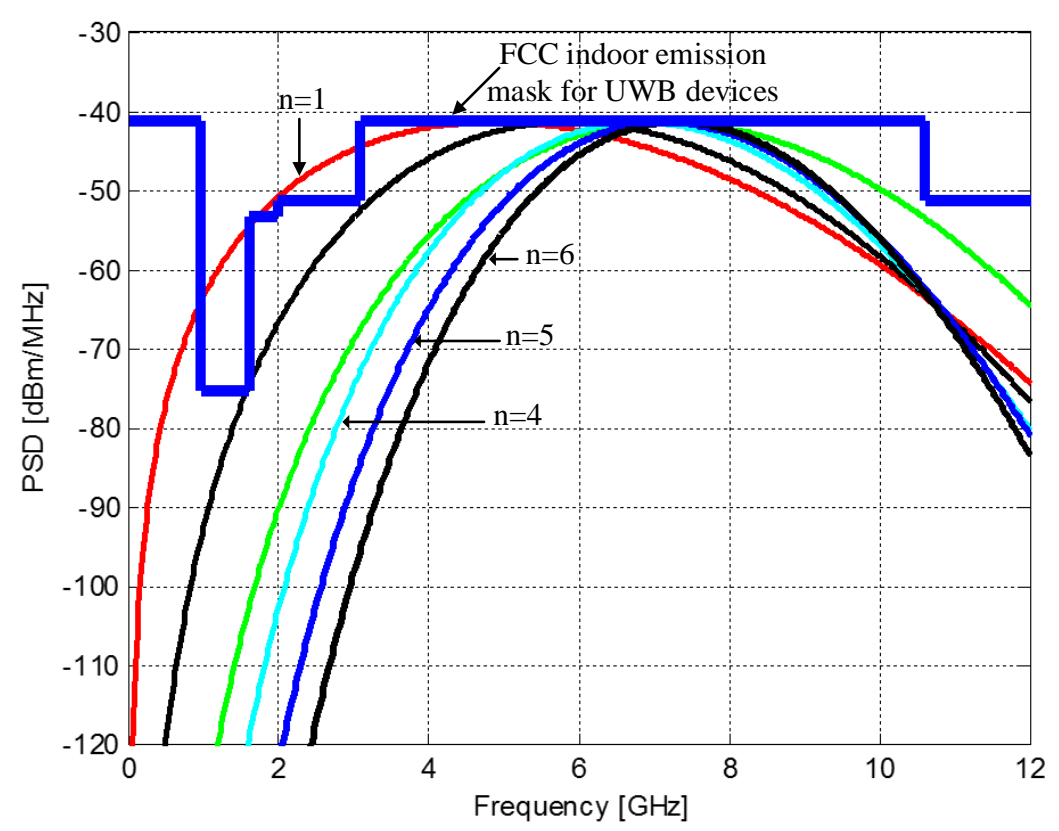

Figure 4. The power spectral density (PSD) of the different derivatives of Gaussian pulses.

It is observed that with impulse radio UWB (IR-UWB) signal, the correlation and therefore the propagation time can be calculated. However, the IR-UWB signal is not strong enough to be processed at the receiver in the case of multiple layers medium (the value of cross-correlation is lower than the noise lever). Hence, an orthogonal pseudo sequence $a(n)$ is applied to the UWB signal in pulse position modulation (PPM) to ensure the cross-correlation is obtained when receiving many reflected signals from many surfaces and buried objects. The conventional UWB-PPM signal with the fourth-order Gaussian monocycle is given as:

$$
s(t)=\sum_{n=1}^{N} g_{4}\left(t-n T_{r}-a_{n} T_{\mathrm{PPM}}\right),
$$

where $T_{r}$ is pulse repetition period and $T_{\mathrm{PPM}}$ is the time shift associated with binary PPM, $a_{n} \in\{0,1\}$, and $N$ is the length of UWB pulses. In this modulation technique, when $a_{n}$ is 0 , there is no additional time shift that modulates the pulse $g_{4}(t)$ and a time shift $T_{\mathrm{PPM}}$ is added to $g_{4}(t)$ when $a_{n}$ is 1 . In our system model, $a_{n}$ does not carry information, it only indicates whether the corresponding pulse is shifted or not.

The propagation distance $d$ in the UWB-PPM system used for positioning the buried object in nondestructive medium is computed by:

$$
d=\frac{c \tau}{2 \sqrt{\varepsilon}}
$$


where $c$ is the velocity of light, and $\tau, \varepsilon$ are the traveling time of UWB pulses and the relative permittivity of the nondestructive medium, respectively.

The traveling time $\tau$ is calculated by the correlation between the received signal from the $i^{\text {th }}$ layer, $r_{i}(t)$, and the template waveform $\omega(t)$ :

$$
R_{i}(\tau)=\int_{-\infty}^{\infty} r_{i}(t) \omega(t) d t
$$

With the conventional UWB-PPM systems, the template waveform at the receiver is $\left(g_{4}(t)-\right.$ $\left.g_{4}\left(t-T_{\mathrm{PPM}}\right)\right)$ and the correlation of this system is denoted by $R_{0 i}$ and has the form:

$$
R_{0 i}(\tau)=\int_{-\infty}^{\infty} r_{i}(t)\left[g_{4}(t)-g_{4}\left(t-T_{\mathrm{PPM}}\right)\right] d t .
$$

Define the autocorrelation function of the fourth-order Gaussian monocycle in (4) as $R_{G 4}(\tau)$, we have

$$
\begin{aligned}
& R_{G 4}(\tau)=\int_{-\infty}^{\infty} g_{4}(t) g_{4}(t-\tau) d t \\
& R_{0 i}(\tau)=R_{G 4}(\tau)-R_{G 4}\left(\tau-T_{\mathrm{PPM}}\right) .
\end{aligned}
$$

The traveling time of UWB signal is the value that makes the correlation function hit its maximum; it corresponds to twice the transmission time from the transceiver to the reflective surface of the $i^{\text {th }}$ layer as shown in Figure 5 and given as

$$
\tau=\arg _{\tau}\left(\max \left(R_{i}\right)\right)
$$

where $\max \left(R_{i}\right)$ is the local extreme value corresponding to the reflected signals from the surface of the layers or object.

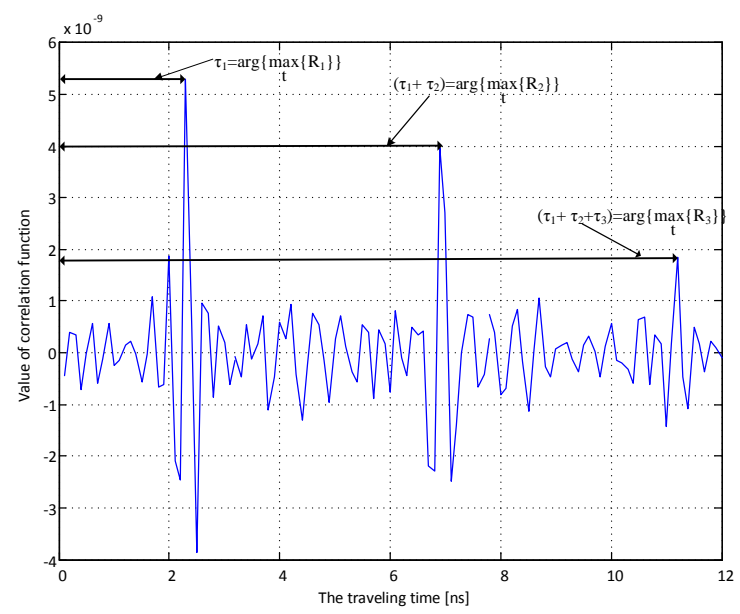

Figure 5. The local extreme values of correlation output corresponding to propagation distances in Figure 1.

According to (6), $\tau$ is a function of $\varepsilon$ and the distance $d$, due to both $d$ and $\varepsilon$ are unknown; they should be estimated by large enough values of $\tau$ using LMFA. To increase the accuracy in the $\tau$ determination procedure, and in estimation of $d, \varepsilon$, the proposed UWB-PPM-ATS technique is applied. 


\section{Proposal of Positioning Approach in Nondestructive Environments}

\subsection{Proposal of UWB-PPM-ATS}

From (1), (5), and (6), it can be seen that the time shift $T_{P P M}$ directly affects the quality of UWB-PPM systems in positioning applications. The performance of the system can be improved by selecting the appropriate value of the time shift. To increase the accuracy in the estimated distance and the position of buried objects by using the UWB-PPM system, the time shift in this system should be selected so that the ability to detect the received UWB pulse is the best. To accomplish this task, in UWB-PPM systems with the time shift $T_{\mathrm{PPM}}$ is invariant, we recommend adjusting this time shift with a certain time constant to achieve its optimal value. The optimal value of $T_{\mathrm{PPM}}$ defined in this paper is the value at which the UWB-PPM system gives the smallest error in distance estimation. So we propose an UWB pulse position modulation with an additional time shift (UWB-PPM-ATS). In the proposed technique, the pulse position will be changed with a time constant denoted by $\zeta$. The signal of UWB-PPM-ATS is given as:

$$
s(t)=\sum_{n=1}^{N} g_{4}\left(t-n T_{r}-a_{n} \cdot\left(T_{P P M}+\zeta\right)\right) .
$$

The "+" sign in (12) means that algebraic additions, so $\zeta$, can take either positive or negative values. The effect of $\zeta$ on the quality of the system will be evaluated later.

An example of the conventional UWB, UWB-PPM, and UWB-PPM-ATS signal shapes are illustrated in Figure 6. In Figure 6, there is an example of a seven pulses sequence carrying seven bits 1010101 . The pulse width is $0.28 \mathrm{~ns}$, and it adopts the fourth derivative of the Gaussian function described by the blue line; the conventional PPM modulated pulses sequence is denoted by the red line. Whereby, when the bit value is 0 , the transmitter will send out a pulse $g_{40}(t)$ as the original pulse (without any change from the unmodulated pulse) when the value bit is 1 , the transmitter will send out a pulse $g_{41}(t)$ which is the original pulse with a time shift of $T_{\mathrm{PPM}}$. With the proposed UWB-PPM-ATS (black line), instead of the normal $T_{\mathrm{PPM}}$ time shift, the new shift level is set to $\left(T_{\mathrm{PPM}}+\zeta\right)$.

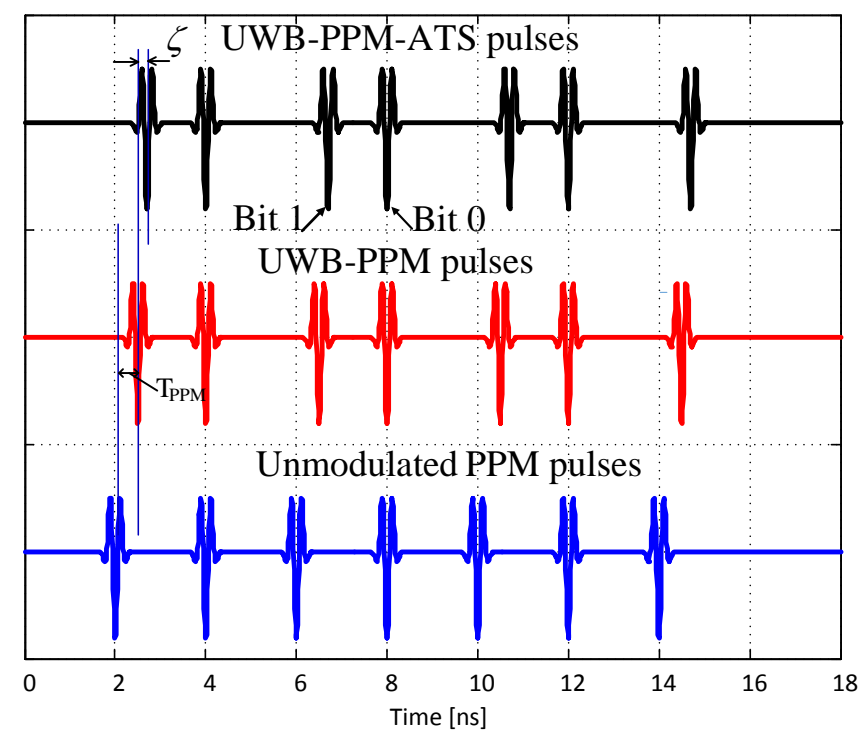

Figure 6. The ultra-wide band (UWB) pulse shapes with pulse position modulation (PPM) and PPM-ATS.

The procedure for estimating distances in UWB-PPM-ATS system is the same as in the conventional system (UWB-PPM) and in (7); the template waveform is $\left(g_{4}(t)-g_{4}\left(t-T_{\mathrm{PPM}}-\zeta\right)\right)$. The correlation of the proposed system denoted by $R_{1 i}$ and determined as: 


$$
R_{1 i}(\tau)=\int_{-\infty}^{\infty} r_{i}(t)\left[g_{4}(t)-g_{4}\left(t-T_{\mathrm{PPM}}-\zeta\right)\right] d t
$$

So, we have:

$$
\left.R_{1 i}(\tau)=R_{G 4}(\tau)-R_{G 4}\left(\tau-T_{\mathrm{PPM}}-\zeta\right)\right] d t .
$$

The shapes of $R_{G 4}(\tau), R_{0 i}(\tau)$ and $R_{1 i}(\tau)$ are shown in Figure 7 .

Figure 7 indicates the autocorrelation function of the fourth-order Gaussian monocycle $R_{G 4}$ and correlation functions of UWB-PPM $R_{0 i}$ and UWB-PPM-ATS $R_{1 i}$ systems (with additional time $\zeta=-0.08 \mathrm{~ns}$ and $\zeta=0.08 \mathrm{~ns}$ ); those functions are compared at the value of $\tau=0$. In the figure below inside Figure 7, the blue line with "+" sign denotes the value of $R_{0 i}(\tau)$, the red line denotes the value of $R_{1 i}(\tau)$ with $\zeta$ gets a negative value of $-0.08 \mathrm{~ns}$, and the dashed black line denotes the value of $R_{1 i}(\tau)$ with $\zeta$ gets a positive value of $0.08 \mathrm{~ns}$. We can observe that the choice of a negative value of $\zeta$ makes $R_{1 i}(\tau)$ get the maximum value at $\tau=0$ and this value is greater than $R_{1 i}(0)$ - with a positive value of $\zeta$ and also $R_{0 i}(0)$ (both of these functions are not maximized at $\tau=0$ ). Thus $\zeta$ will be selected that makes the value of $R_{G 4}\left(\tau-T_{\mathrm{PPM}}-\zeta\right)$ reaches to the minimum point of the $R_{G 4}$ function which is denoted by $R \tau_{\text {opt }}$ as shown in Figure 7. It is clear that, in the UWB-PPM-ATS scheme, the negative values of $\zeta$ give the better correlation function than positive values. The variation of the correlation function with the different of $\zeta$ values is illustrated in Figure 8. In Figure 8, the different values of $\zeta$ lead to the different shapes of the correlation function; the optimal value of $\zeta$ in this case is $\zeta_{\text {opt }}=-0.08 \mathrm{~ns}$ which makes $R_{1 i}(0)$ have the maximum value. When the magnitude of the $\zeta$ increases close to the pulse width ( $\zeta=-0.16 \mathrm{~ns},-0.25 \mathrm{~ns}$ in Figure 8$)$, the corresponding correlation functions will not reach the global maximum near the value of $\tau=0$ and its local maximum points have the values close to the global maximum. This leads to the higher error in determining the global maximum point of these functions. Therefore, depending on the specific parameter configuration of each UWB-PPM system, the value of $\zeta$ will be chosen such that $R_{1 i}(0)$ in the (14) has the largest value.
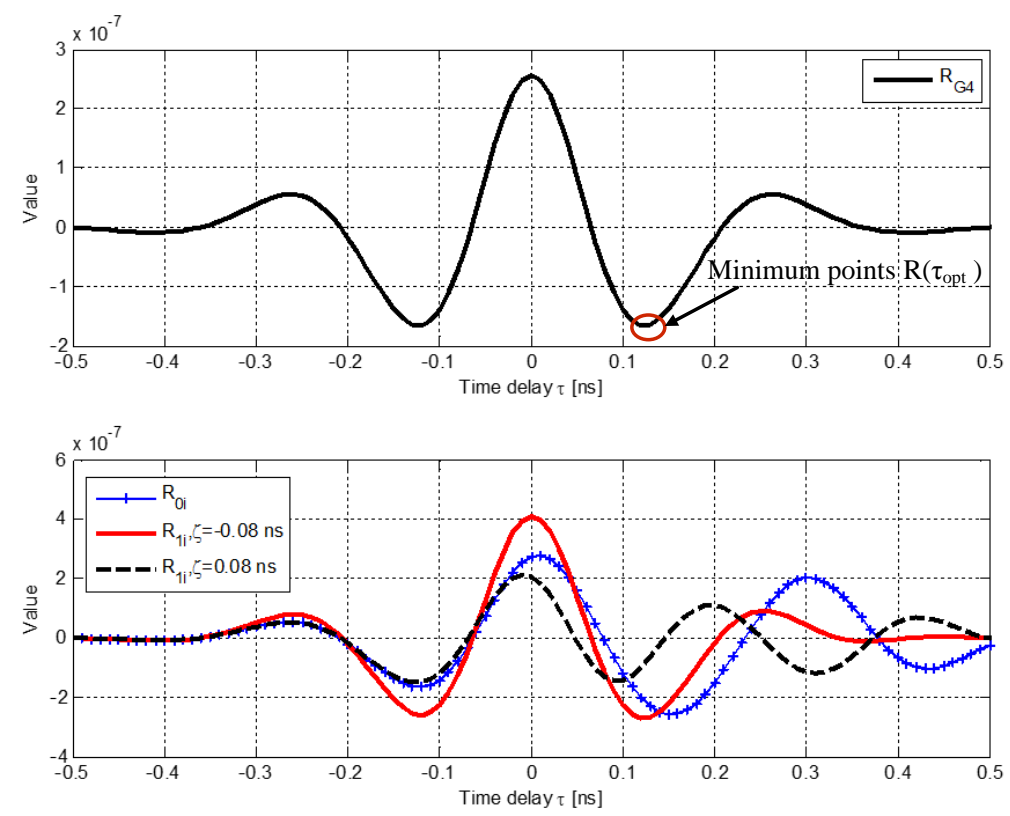

Figure 7. Correlation functions of the conventional UWB-PPM and the proposed UWB-PPT-ATS systems with different time shift. 


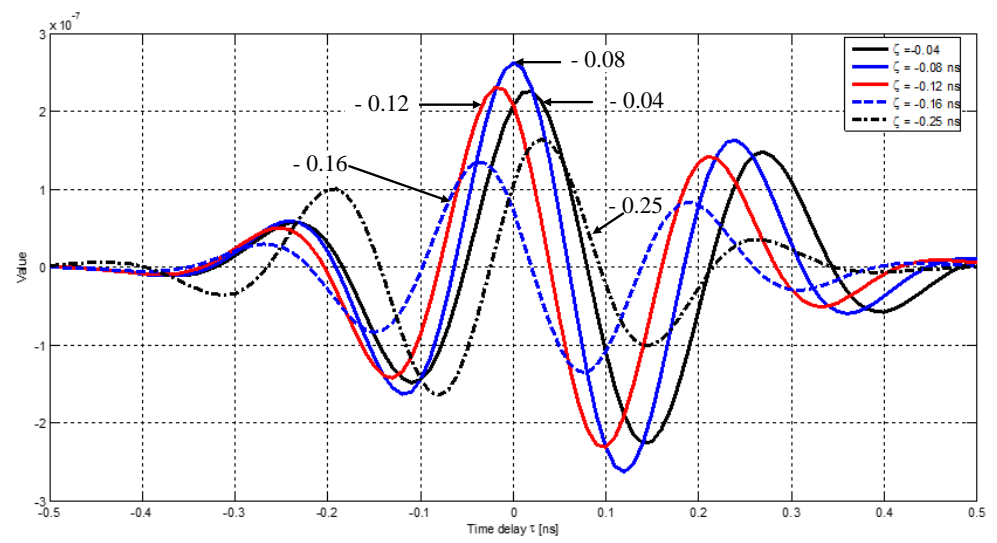

Figure 8. Correlation functions of proposed UWB-PPM-ATS with different additional time shift.

The procedure for estimating distances is based on the traveling time mentioned above and can be applied to a well-known propagation environment; however, it is unavailable for an unknown propagation environment. Consequently, we propose to apply LMFA [28] into the calculation of distances, relative permittivities, and determination of buried object location (depth and horizontal direction) in the case of the unknown propagation environment. The proposed method comes from the fact that the traveling time depends on the relative permittivities, buried object location, and transceiver position. So in order to find the value of these parameters, we change the position of the receiver horizontally and calculate the traveling time corresponding to each position of the transceiver. Based on the transceiver location and computed traveling time, the remaining parameters will be estimated.

\subsection{Estimation Algorithm}

Let us consider a specific system model of UWB-PPM systems illustrated in Figure 9, in which the environment is assumed to have a nondestructive structure that has two layers with relative permittivities of $\varepsilon_{1}$ and $\varepsilon_{2}$.

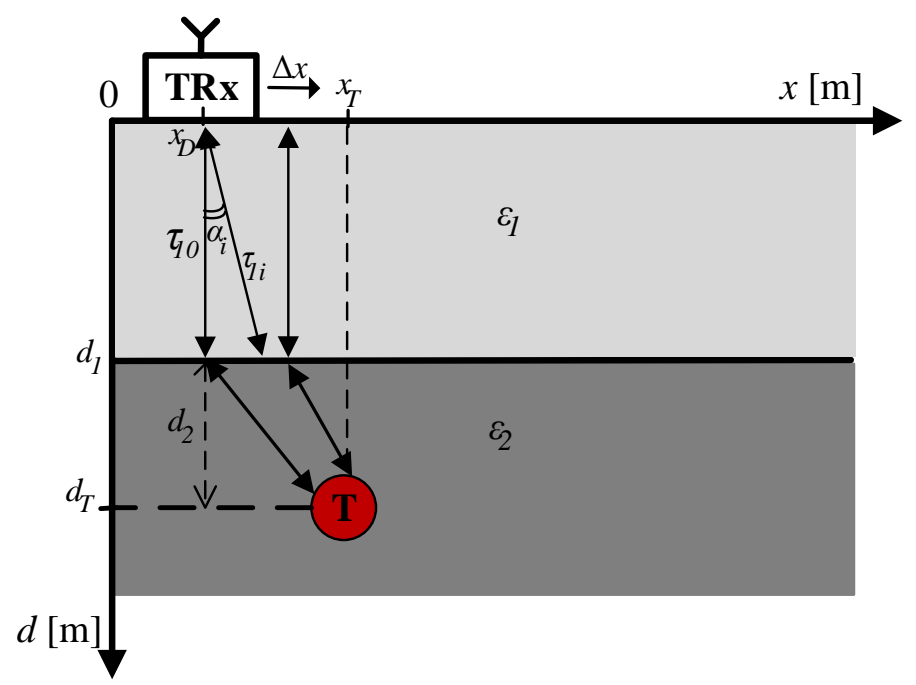

Figure 9. Target system model in an environment with nondestructive structure of two layers.

To determine the environmental parameters and also the location of the target " $\mathrm{T}$ ", the transceiver generates UWB pulses with rotation angles of the transceiver antenna are $\alpha_{i}$ and get the reflected pulses. 
The traveling time $\tau_{1 i}$ corresponding to the $i^{\text {th }}$ time of emission is calculated according to (11), the geometric relationship between $\tau_{1 i}, d_{1}$, and $\varepsilon_{1}$ is indicated in (15).

$$
\tau_{1 i}=\frac{2 d_{1} \sqrt{\varepsilon_{1}}}{c \cdot \cos \alpha_{i}}
$$

where $\alpha_{i}$ is the rotation angle of antenna and:

$$
\alpha_{i}=i . \Delta \alpha
$$

where $\Delta \alpha$ is the minimum rotation angle of the transceiver antenna. Thus the values of $\varepsilon_{1}$ and $d_{1}$ in (15) are estimated based on known pairs $\left(\alpha_{i}, \tau_{1 i}\right)$. Then, to locate buried objects, the device is moved horizontally, and the transmitter emits pulses in the perpendicular direction after every movement step of $\Delta x$. Denote $\tau_{2 i}$ is the traveling time of the transmitted signals, reflected from the buried object and returned to the transceiver at position of $x_{D i}$, it is computed by:

$$
\tau_{2 i}=\tau_{10}+\frac{\sqrt{\varepsilon_{2}\left(d_{2}^{2}+\left(x_{T}-x_{D i}\right)^{2}\right)}}{c},
$$

where $x_{T}$ is the horizontal coordinates of the buried object and $\tau_{10}$ is the traveling time of reflected signal at the interface between two layers of the environment in $d$ direction. $\tau_{2 i}$ also computed according to (11), the values of $\varepsilon_{2}, d_{2}$, and $x_{T}$ in (17) are estimated based on known pairs $\left(\tau_{2 i}, \tau_{10}, x_{D i}\right)$, and

$$
x_{D i}=i . \Delta x .
$$

The proposed algorithm to estimate the values of those parameters is indicated in Figure 10 and described in detail as below

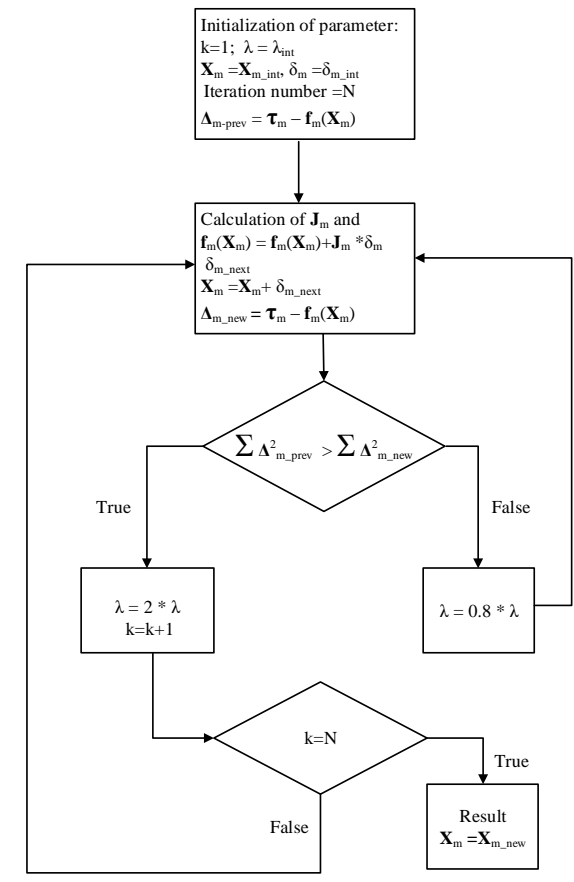

Figure 10. Flowchart of estimated algorithms.

In Figure 10, the unknown parameter vectors are denoted by:

$$
\mathbf{X}_{1}=\left(\widehat{\varepsilon}_{1}, \widehat{d}_{1}\right) ; \mathbf{X}_{2}=\left(\widehat{\varepsilon}_{2}, \widehat{d}_{2}, \widehat{x}_{T}\right),
$$


and the index $m$ in Figure 10 is used to replace for 1, 2. With the known pairs $\left(\tau_{1 i}, \alpha_{i}\right)$ and $\left(\tau_{2 i}, x_{D i}\right)$, the unknown parameter vectors $\mathbf{X}_{1}$ and $\mathbf{X}_{2}$ are determined such that the sum of the squares of the deviations $S_{i}\left(\mathbf{X}_{i}\right)$ are minimized:

$$
\begin{aligned}
& X_{1}=\arg \min _{\mathbf{X}_{1}} S_{1}\left(\mathbf{X}_{1}\right)=\arg \min _{\mathbf{X}_{1}} \sum_{i=1}^{M}\left[\tau_{1 i}-f_{1}\left(\alpha_{i}, \mathbf{X}_{1}\right)\right]^{2}, \\
& X_{2}=\arg \min _{\mathbf{X}_{2}} S_{2}\left(\mathbf{X}_{2}\right)=\arg \min _{\mathbf{X}_{2}} \sum_{i=1}^{M}\left[\tau_{2 i}-f_{2}\left(x_{D i}, \mathbf{X}_{2}\right)\right]^{2},
\end{aligned}
$$

where $f_{1}\left(\alpha_{i}, \mathbf{X}_{1}\right)$ and $f_{2}\left(x_{D i}, \mathbf{X}_{2}\right)$ are:

$$
\begin{aligned}
f_{1}\left(\alpha_{i}, \mathbf{X}_{1}\right) & =\frac{\widehat{d_{1} \cdot \sqrt{\widehat{\varepsilon}_{1}}}}{c \cdot \cos \alpha_{i}}, \\
f_{2}\left(x_{D i}, \mathbf{X}_{2}\right) & =\frac{\sqrt{\widehat{\varepsilon}_{2}\left(\widehat{d}_{2}^{2}+\left(\widehat{x}_{T}-x_{D i}\right)^{2}\right)}}{c},
\end{aligned}
$$

where. denotes the estimated values of the parameters; the vectors $\mathbf{X}_{\mathbf{1}}$ and $\mathbf{X}_{\mathbf{2}}$ were estimated according to the steps as shown in Figure 10 as follows:

+ Assign to $\mathbf{X}_{1}, \mathbf{X}_{2}$ any arbitrary initialization values, denoted by $\mathbf{X}_{1-i n t}, \mathbf{X}_{2-i n t}$;

+ In each iteration step, the parameter vectors $\mathbf{X}_{1}, \mathbf{X}_{2}$ are replaced by new estimates $\left(\mathbf{X}_{1}+\delta_{1}\right),\left(\mathbf{X}_{2}\right.$ $+\delta_{1}$ ) with $\delta_{1}, \delta_{2}$ are updated step vectors and we have:

$$
\begin{aligned}
\mathbf{S}_{1}\left(\mathbf{X}_{1}+\delta_{1}\right) \approx & {\left[\tau_{1}-\mathbf{f}_{1}\left(\mathbf{X}_{1}\right)\right]^{T}\left[\tau_{1}-\mathbf{f}_{1}\left(\mathbf{X}_{1}\right)\right] } \\
& -2\left[\tau_{1}-\mathbf{f}_{1}\left(\mathbf{X}_{1}\right)\right]^{T} \mathbf{J}_{1} \delta_{1}+\delta_{1}^{T} \mathbf{J}_{1}^{T} \mathbf{J}_{1} \delta_{1}, \\
\mathbf{S}_{2}\left(\mathbf{X}_{2}+\delta_{2}\right) \approx & {\left[\mathbf{x}_{D}-\mathbf{f}_{2}\left(\mathbf{f}_{2}\right)\right]^{T}\left[\mathbf{x}_{D}-\mathbf{f}_{2}\left(\mathbf{X}_{2}\right)\right] } \\
& -2\left[\mathbf{x}_{D}-\mathbf{f}_{2}\left(\mathbf{X}_{2}\right)\right]^{T} \mathbf{J}_{2} \delta_{2}+\delta_{2}^{T} \mathbf{J}_{2}^{T} \mathbf{J}_{2} \delta_{2},
\end{aligned}
$$

with $\mathbf{J}_{1,2}$ is the Jacobian matrix, whose $i^{\text {th }}$ row equals $\mathbf{J}_{1 i}$ and $\mathbf{J}_{2 i}$, respectively:

$$
\begin{gathered}
\mathbf{J}_{1 i}=\frac{\partial f_{1}\left(\alpha_{i}, \mathbf{X}_{1}\right)}{\partial \mathbf{X}_{1}}, \\
\mathbf{J}_{2 i}=\frac{\partial f_{2}\left(x_{D i}, \mathbf{X}_{2}\right)}{\partial \mathbf{X}_{2}} .
\end{gathered}
$$

To get the minimum value of the sums $\mathbf{S}_{1}, \mathbf{S}_{2}$, the update step vectors $\delta_{1,2}$ is calculated such that the derivative of $\mathbf{S}_{1,2}\left(\mathbf{X}_{1,2}+\delta_{1,2}\right)$ with respect to $\delta_{1,2}$ has a result of zero, so, $\delta_{1,2}$ can be determined satisfying:

$$
\begin{array}{r}
{\left[\mathbf{J}_{1}^{T} \mathbf{J}_{1}+\lambda \operatorname{diag}\left(\mathbf{J}_{1}^{T} \mathbf{J}_{1}\right)\right] \delta_{1}=\mathbf{J}_{1}^{T}\left[\tau_{1}-\mathbf{f}_{1}\left(\mathbf{X}_{1}\right)\right],} \\
{\left[\mathbf{J}_{2}^{T} \mathbf{J}_{2}+\lambda \operatorname{diag}\left(\mathbf{J}_{2}^{T} \mathbf{J}_{2}\right)\right] \delta_{2}=\mathbf{J}_{2}^{T}\left[\mathbf{x}_{D}-\mathbf{f}_{2}\left(\mathbf{X}_{2}\right)\right],}
\end{array}
$$

where the damping factor $\lambda$ (non-negative) is adjusted at each iteration. If $\mathbf{S}_{1,2}$ is reduced rapidly, a smaller value of $\lambda$ can be used, whereas if in an iteration does not reduce the residual, $\lambda$ can be increased. The update step vectors are computed as follows:

$$
\begin{gathered}
\delta_{1}=\left[\mathbf{J}_{1}^{\mathrm{T}} \mathbf{J}_{1}+\lambda \operatorname{diag}\left(\mathbf{J}_{1}^{T} \mathbf{J}_{1}\right)\right]^{-1} \mathbf{J}_{1}^{T}\left[\tau_{1}-\mathbf{f}_{1}\left(\mathbf{X}_{1}\right)\right], \\
\delta_{2}=\left[\mathbf{J}_{2}^{T} \mathbf{J}_{2}+\lambda \operatorname{diag}\left(\mathbf{J}_{2}^{T} \mathbf{J}_{2}\right)\right]^{-1} \mathbf{J}_{2}^{T}\left[\mathbf{x}_{D}-\mathbf{f}_{2}\left(\mathbf{X}_{2}\right)\right] .
\end{gathered}
$$

After a certain number of iterations, the output of LMFA is the final estimated values of system parameters that meet the constraint condition in (21), as shown in Figure 10. 


\section{Simulation Results and Discussion}

\subsection{Simulation Parameters}

The accuracy of methods for determining the distance and characteristics of a multilayered reflective environments using UWB pulses is strongly dependent on UWB signal processing techniques. UWB-PPM is one of the candidates for positioning technology in a nondestructive environment with multiple reflective layers; with the proposed UWB-PPM-ATS, our trials indicated that the UWB-PPM pulses shifted with a certain time constant can be used to improve the precision in estimating the distance. All the numerical results in this paper were computed using Matlab.

First, we compare the exact estimation errors of the UWB-OOK, UWB-PPM and UWB-PPM-ATS systems with the results obtained from the simulations and the actual value of the parameters use (6), (8), (11), (13) and (30). Here OOK is one of the UWB pulse amplitude modulation techniques and has two level modulation with the bits are 0 and 1 . When sending a bit 0 , the transmitter will not send anything. When sending a bit 1 , the transmitter will send a pulse [29], and the template waveform $\omega(t)$ at the receiver of UWB-OOK system is $g_{4}(t)$. In addition, the performance of UWB-OOK, UWB-PPM and UWB-PPM-ATS systems were evaluated for an environment with known characteristics (for example, here are three layers as indicated in Figure 1). Finally, the location determination technique in a unknown environment with multiple reflection layers using the LMFA based on the estimated parameters of those UWB systems is presented with the system model as shown in Figure 9. The comparisons between the above systems are evaluated in term of errors between the estimated values from the considered system and the true values. Based on the PSD plot of the Gaussian impulses shown in Figure 4, most of the numerical results presented in this section are based on analysis using the fourth-order Gaussian monocycle. The parameters of the example UWB systems are listed in Table 1 and follow [5].

To assess the performance of those UWB systems, the error of estimated distance is defined as follows:

$$
\delta_{d}=\left|\hat{d}_{i}-d_{t r i}\right|
$$

where $\hat{d}_{i}$ denotes the estimated distance and $d_{t r i}$ is the true value.

Table 1. Simulation parameters.

\begin{tabular}{ccc}
\hline Parameter & Notation & Value \\
\hline Time normalization factor & $\mu_{p}$ & $0.2877 \mathrm{~ns}$ \\
Transmitted power & $P_{T x}$ & $-35.4 \mathrm{~dB}$ \\
The amplitude factors & $A_{1}, A_{2}, A_{3}$ & $0.33,0.13,0.14$ \\
Number of pulses & $N$ & 100 \\
Noise power & $N_{0} / 2$ & $-102 \mathrm{~dB}$ \\
Time shift of PPM & $T_{P P M}$ & $0.2 \mathrm{~ns}$ \\
Additional time shift & $\zeta$ & $-0.08 \mathrm{~ns},-0.16 \mathrm{~ns}$ \\
Relative permittivity & & $4 ; 3 ; 5$ \\
with heterogeneous medium & $\varepsilon_{1}, \varepsilon_{2}, \varepsilon_{3}$ & \\
\hline
\end{tabular}

In Figure 1, without generality, we assume that the propagation environment is heterogeneous with three layers: sand (dry), sandy soil (dry) and granite (dry) and the relative permittivities $\varepsilon_{1}, \varepsilon_{2}, \varepsilon_{3}$ are $4,3,5$, respectively. Those layers are assumed to be dry to reduce the attenuation of environment, so propagation velocity in those layers are $15 \mathrm{~cm} / \mathrm{ns}, 17.32 \mathrm{~cm} / \mathrm{ns}, 13.42 \mathrm{~cm} / \mathrm{ns}$ and the attenuation is $0.01 \mathrm{~dB} / \mathrm{m}$ [30]. With a known transmission environment, those distance can be estimated using (6), where $\tau_{i}$ is computed according to (8), (11) and (13). Then the errors of estimated distances are calculated according to (30) and illustrated in Figure 11 for OOK, PPM, ATS systems with $\zeta=-0.08$ ns and $\zeta=-0.16$ ns. In Figure 11, the black line denotes the average error of the OOK 
system, the blue line denote the error of the PPM system and the red lines denote the error of the ATS system, all with the same system parameters. Observe that the relative error of the UWB-OOK system is about $24 \%$, of UWB-PPM is about $11 \%$, of UWB-PPM-ATS with $\zeta=-0.08 \mathrm{~ns}$ is about $7 \%$, with $\zeta=-0.16 \mathrm{~ns}$ is about $13 \%$; so the UWB-PPM- ATS with $\zeta=-0.08$ ns performs better for all distance values. This can be explained by comparing the correlation functions which are given in (8), (13) and Figures 7 and 8; we observe that $R_{1 i}(0)$ is significantly greater than $R_{0 i}(0)$ for $\zeta=-0.08 \mathrm{~ns}$ and $R_{0 i}(0)$ is not the maximum value of $R_{0 i}(\tau)$, which leads to a smaller than average error in the PPM-ATS with $\zeta=-0.08$ ns when compared to PPM scheme; but with $\zeta=-0.16 \mathrm{~ns}, R_{1 i}(0)$ is less than $R_{0 i}(0)$ thus the PPM outperforms the PPM-ATS. Besides that, the UWB-OOK is one of the amplitude modulation techniques, thus it is affected by the transmission environment and more difficult to separate the received signal from noise interference in comparison with the PPM techniques which have a constant amplitude. The results in Figure 11 indicate that the time shift $\zeta$ directly affects the performance of PPM systems. The value of $\zeta$ is selected so that $\left(T_{\mathrm{PPM}}+\zeta\right)$ achieves the optimal value at which $R_{G 4}\left(\tau-T_{\mathrm{PPM}}-\zeta\right)$ gets the minimum value. From (6) and (13), we also know that $\zeta$ is determined by the pulse shape and the pulse width normalization factor $\mu_{p}$. Due to the influence of the UWB-PPM system to the choice of the time shift, a suitable value of $\zeta$ should be chosen according to the specific pulse shape and pulse width employed in a particular UWB application.

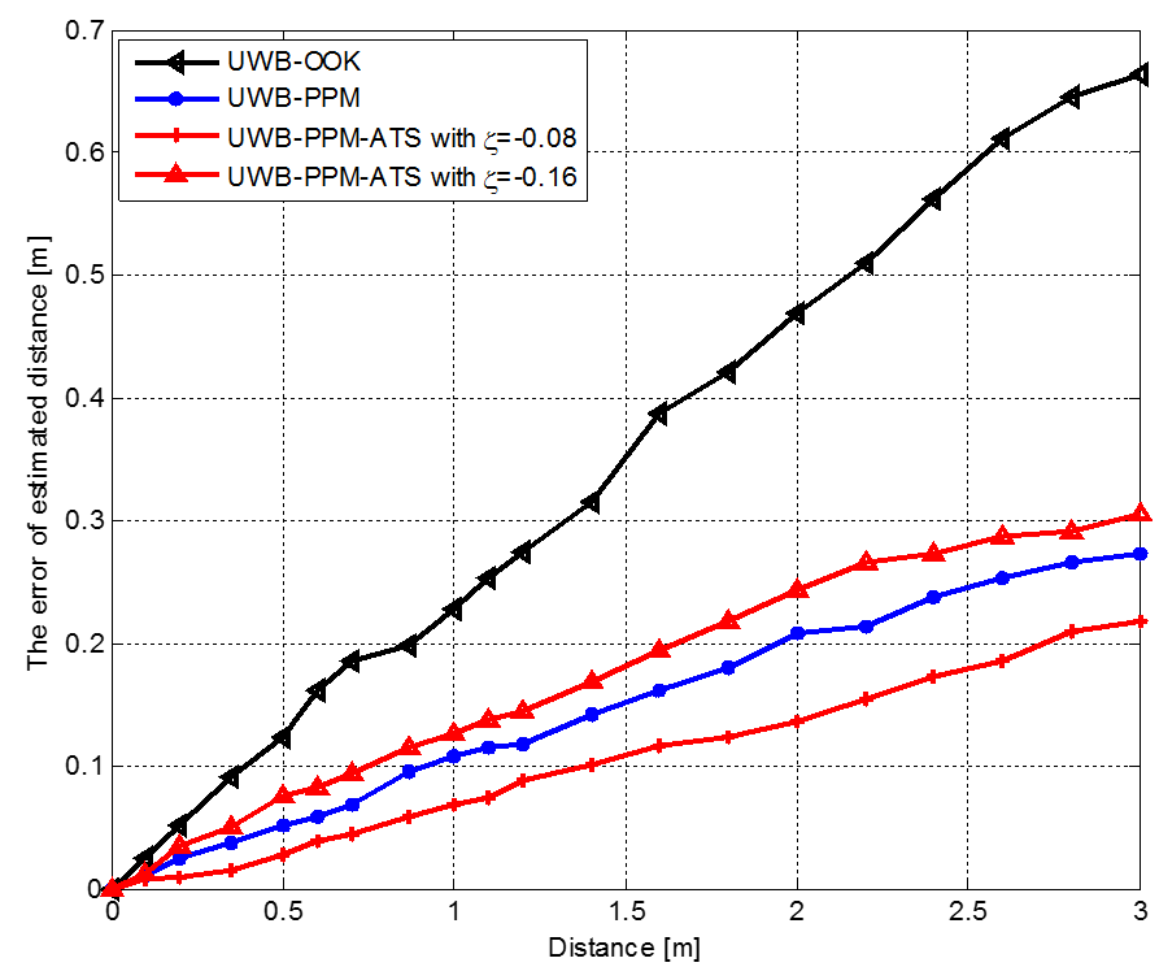

Figure 11. Comparison of distance estimation errors between OOK, PPM and the proposed UWB-PPM-ATS modulation techniques.

\subsection{Determine the Location of Buried Object by LMFA}

As mentioned above, the propagation distance can only be determined using the correlation function if the characteristic of the environment is well-known; however, this is impossible with unknown environment. Moreover, the position of buried object cannot be determined by only the propagation distances; it must be determined in both $x$ and $d$ directions $\left(x_{T}, d_{T}\right.$ in Figure 9). The parameters of each layer in the system model in Figure 9 are estimated sequentially using LMFA as presented in Section 3.2. The parameters of system model and the initialization vectors of the LMFA algorithm are listed in Table 2 in which two layers of environment are assumed to be dry sand and concrete with the corresponding propagation velocity of $16 \mathrm{~cm} / \mathrm{ns}$ and $14.14 \mathrm{~cm} / \mathrm{s}$. 
Table 2. Initialization parameters of the model.

\begin{tabular}{ccc}
\hline Parameter & Notation & Value \\
\hline The thickness of the 1st layer & $d_{1}$ & $0.63 \mathrm{~m}$ \\
Depth of ' $\mathrm{T}$ ' in the 2nd layer & $d_{2}$ & $0.42 \mathrm{~m}$ \\
X-coordinate of ' $\mathrm{T}$ ' & $x_{T}$ & $1.6 \mathrm{~m}$ \\
Relative permittivity & $\varepsilon_{1}, \varepsilon_{2}$ & $3.5,4.5$ \\
Damping factor & $\lambda$ & 3 \\
Movement step of the device & $\Delta x$ & $20 \mathrm{~cm}$ \\
\hline
\end{tabular}

First the parameters $\varepsilon_{1}, d_{1}$ are estimated based on the propagation time $\tau_{1 i}$ and rotation angle $\alpha_{i}$ with both UWB-PPM and UWB-PPM-ATS systems. Then, in a similar way, the parameters $d_{2}, \varepsilon_{2}$, and $x_{T}$ are estimated based on $\boldsymbol{\sigma}_{2}$ and $\mathbf{x}_{\mathbf{D}}$; therefore, the depth of buried object $d_{T}=d_{1}+d_{2}$ is also determined. The estimated results after 30 iterations and errors of UWB systems are listed in Table 3 and Figures 12-14. Figures 12 and 13 show the relationship between $\tau_{1 i}$ and $\alpha_{i}$ in (15). In those figures, the dashed black line denote the curve of $\tau_{1 i}$ vs. $\alpha_{i}$ with the true values of $d_{1}$ and $\varepsilon_{1}$; the blue dots are $\tau_{1 i}$ values measured by simulated UWB systems and the red line denote the curve of $\tau_{1 i}$ vs. $\alpha_{i}$ with the estimated values $\widehat{d_{1}}$ and $\widehat{\varepsilon_{1}}$. Figure 14 shows the curve of $\left(\tau_{2 i}-\tau_{10}\right)$ depends on $x_{D i}$ according to (17). In those figures, the dashed black line denotes the curve of $\left(\tau_{2 i}-\tau_{10}\right)$ vs. $x_{D i}$ with the true values of $d_{2}, \varepsilon_{2}$ and $x_{T}$; the blue dots are $\left(\tau_{2 i}-\tau_{10}\right)$ values measured by simulated UWB systems and the red line denotes the curve of $\left(\tau_{2 i}-\tau_{10}\right)$ vs. $x_{D i}$ with the estimated values $\widehat{d_{2}}, \widehat{\varepsilon_{2}}$ and $\widehat{x_{T}}$.
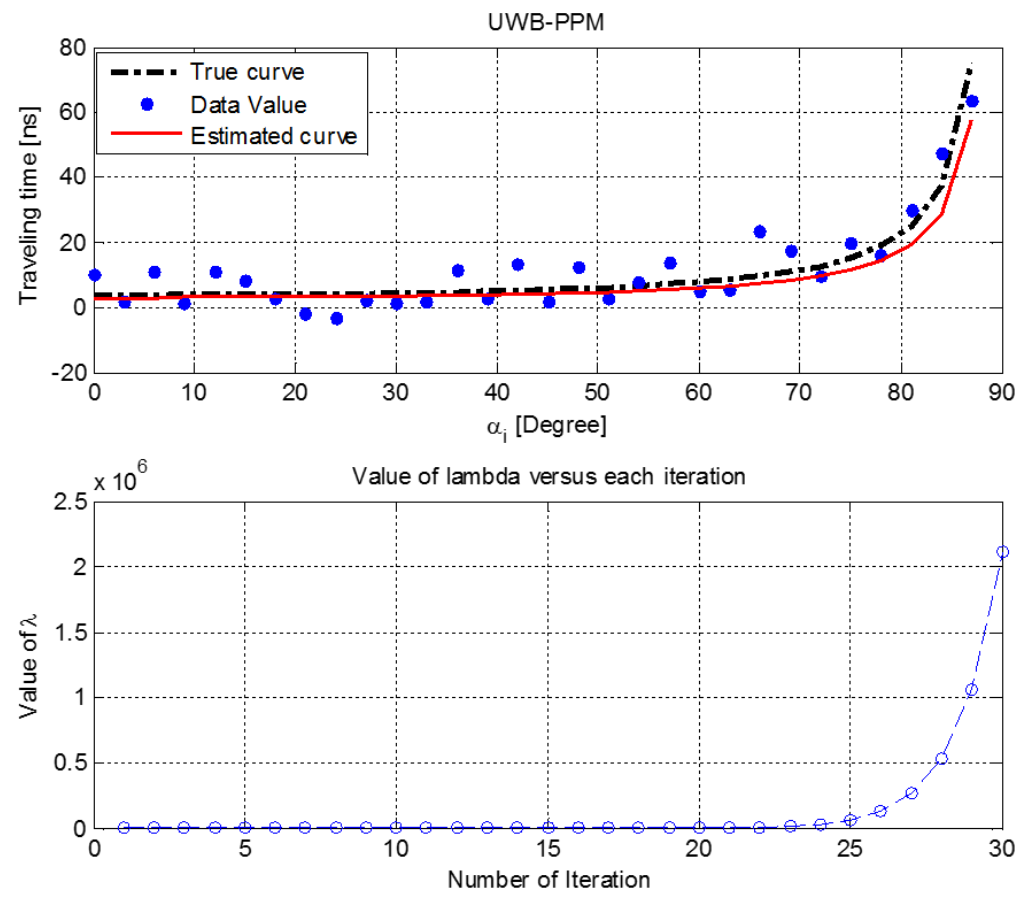

Figure 12. The results of estimating $d_{1}$ and $\varepsilon_{1}$ of the conventional UWB-PPM based on Levenberg-Marquardt Fletcher algorithm (LMFA). 

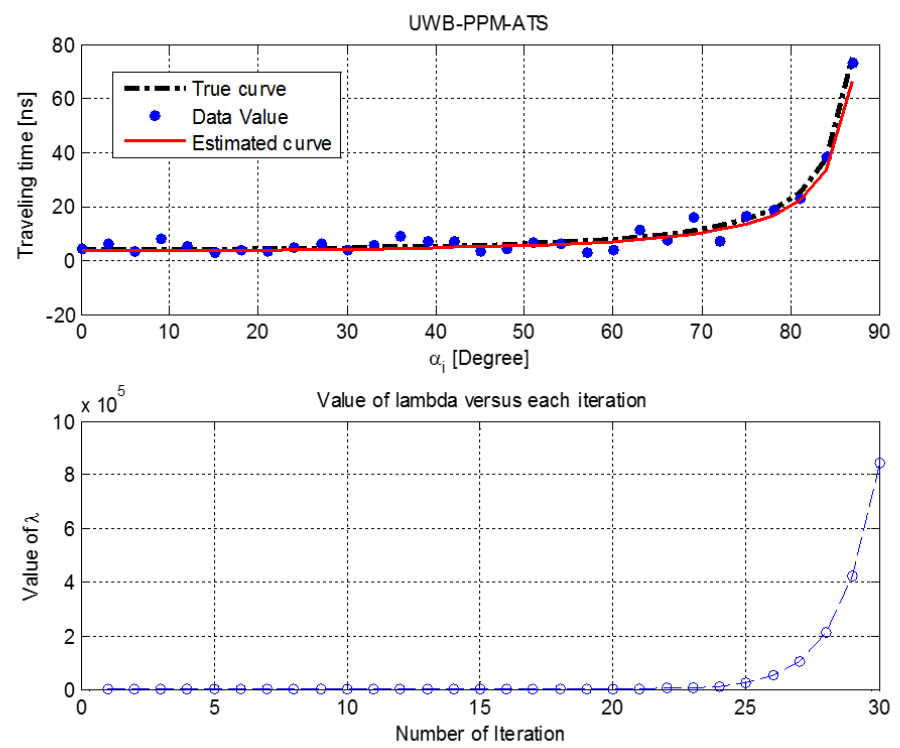

Figure 13. The results of estimating $d_{1}$ and $\varepsilon_{1}$ of the proposed UWB-PPM-ATS based on LMFA.
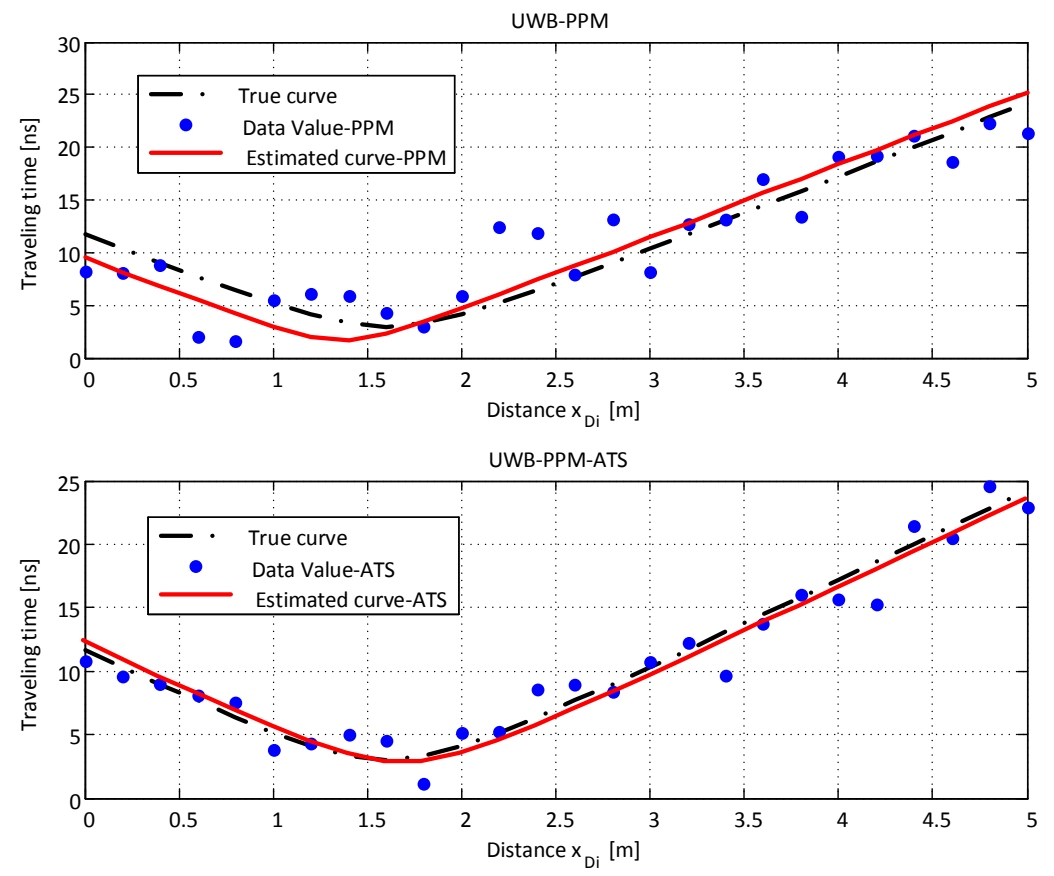

Figure 14. The results of estimating $d_{2}, \varepsilon_{2}$ and $x_{T}$ of the conventional UWB-PPM and the proposed UWB-PPM-ATS based on LMFA.

Table 3 and Figure 15 show the estimated results by the UWB systems. As seen, the modulation techniques for UWB pulses combine with nonlinear estimation method LMFA can be used to determine the thickness of different layers in the nondestructive environments, their relative permittivities and also the position of buried object. We observe that the UWB-PPM-ATS system outperforms the UWB-PPM system for all parameters of the model. This behavior results directly from the features of the correlation functions of different signals shown in Figures 7 and 8. When using UWB-PPM-ATS technique with the optimal value of $\zeta$, the estimated traveling time values have a smaller error than using UWB-PPM technique, so the results from the LMFA of the PPM-ATS system give the higher accuracy than conventional PPM system. 
Table 3. The results of the estimated parameters.

\begin{tabular}{cccccc}
\hline Parameter/Notation & $\boldsymbol{d}_{\mathbf{1}}$ & $\boldsymbol{d}_{\mathbf{2}}$ & $\boldsymbol{x}_{\boldsymbol{T}}$ & $\boldsymbol{\varepsilon}_{\mathbf{1}}$ & $\varepsilon_{2}$ \\
\hline True value [m] & 0.63 & 0.42 & 1.6 & 3.5 & 4.5 \\
By UWB-OOK [m] & 0.9418 & 0.6623 & 2.1362 & 4.3216 & 5.7261 \\
By UWB-PPM [m] & 0.7923 & 0.5742 & 1.9316 & 2.7109 & 3.3590 \\
By UWB- & & & & & \\
PPM-ATS [m] & 0.6523 & 0.3899 & 1.7107 & 3.2829 & 4.8167 \\
Error of UWB-OOK & 0.3118 & 0.2423 & 0.5362 & 0.8216 & 1.2261 \\
Error of UWB-PPM & 0.1623 & 0.1542 & 0.3316 & 0.7891 & 1.141 \\
$\quad$ Error of & & & & & \\
UWB-PPM-ATS & 0.0223 & 0.0301 & 0.1107 & 0.2171 & 0.3167 \\
\hline
\end{tabular}

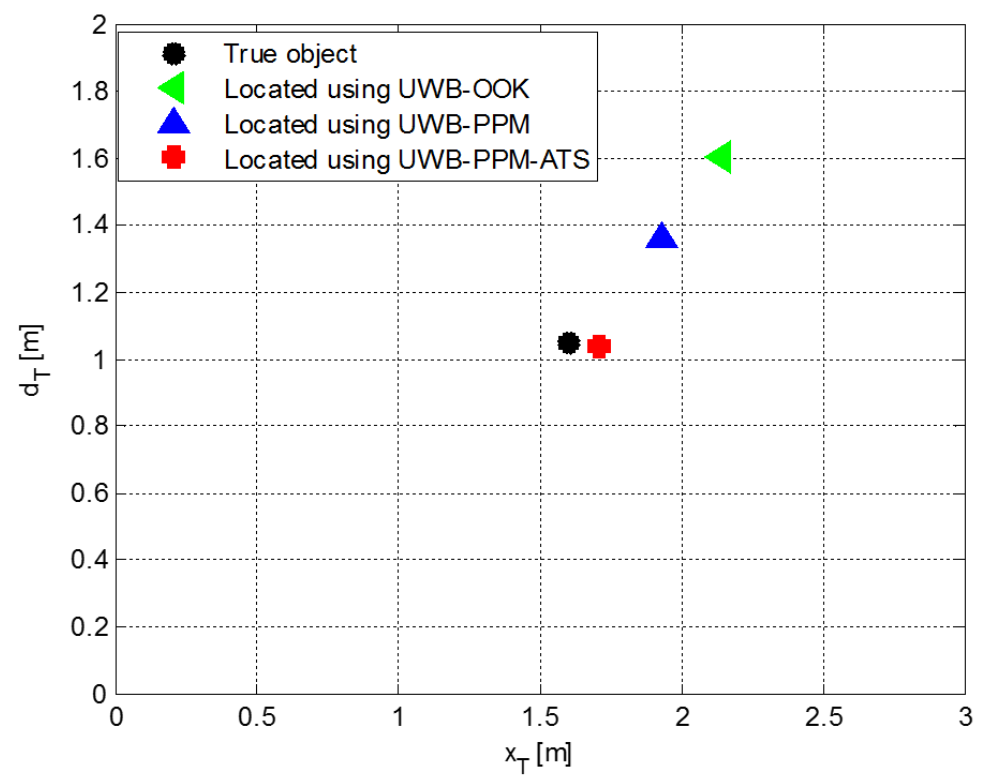

Figure 15. The true and estimated locations of buried object using the conventional UWB-PPM and the proposed UWB-PPM-ATS.

\section{Conclusions}

In this paper, the authors have proposed an UWB pulse position modulation with an additional time-shift technique for positioning applications in nondestructive environments of the UWB systems. The proposed method is utilized to determine the distances and the position of a buried object in heterogeneous environments. The proposed technique's quality is assessed based on the calculation errors compared to the actual values and comparison with the conventional modulation methods. Combining with the Levenberg-Marquardt Fletcher algorithm to determine the buried object location when the propagation environment is unknown, the efficiency of the proposed technique is better than the PPM-UWB system with the same model. Evaluating different UWB systems based on the calculation of errors provides reliable information for choosing modulation schemes with suitable parameters in the design of UWB systems for positioning applications, especially in nondestructive environments.

However, in this work, only one buried object location is determined; multiple buried object estimation algorithm will be researched in our future works. Additionally, the research on parameters of the buried object, such as shape, size, permittivity and so on, is left for the future work.

Author Contributions: Conceptualization, N.T.H. and P.T.H.; methodology, N.T.H. and N.L.C.; software, N.T.H.; validation, N.T.H. and P.T.H.; formal analysis, P.T.H. and N.L.C.; investigation, N.T.H.; resources, N.T.H.; data curation, P.T.H.; writing-original draft preparation, N.T.H.; writing-review and editing, N.T.H. and N.L.C.; visualization, N.L.C.; supervision, P.T.H.; project administration, P.T.H. All authors have read and agreed to the published version of the manuscript. 
Funding: This research received no external funding.

Conflicts of Interest: The authors declare that there is no conflict of interest regarding the publication of this paper.

\section{Abbreviations}

The following abbreviations are used in this manuscript:

$\begin{array}{ll}\text { UWB } & \text { Ultra-wide band } \\ \text { UWB-PPM } & \text { Ultra-wide band pulse position modulation } \\ \text { UWB-OOOK } & \text { Ultra-wide band On-Off Keying } \\ \text { UWB-PPM-ATS } & \text { Ultra-wide band pulse position modulation with optimized additional time shift } \\ \text { LMFA } & \text { Levenberg-Marquardt Fletcher algorithm }\end{array}$

\section{References}

1. Goyal, V. Pulse generation and analysis of ultra wide band system model. GESJ Comput. Sci. Telecommun. 2012, 2, 3-6. ISSN 1512-1232.

2. U. F. C. Commission. Revision of Part 15 of the Commission's Rules Regarding Ultra-Wideband Transmission Systems; First report and order, technical report, Feb, Tech. Rep.; FCC: Washington, DC, USA, 2002.

3. Goyal, V.; Dhaliwal, B. Optimal pulse generation for the improvement of ultra wideband system performance. In Proceedings of the 2014 Recent Advances in Engineering and Computational Sciences (RAECS), Chandigarh, India, 6-8 March 2014; IEEE: Piscataway, NJ, USA, 2014; pp. 1-6.

4. Welborn, M.L. System considerations for ultra-wideband wireless networks. In Proceedings of the RAWCON 2001, 2001 IEEE Radio and Wireless Conference (Cat. No. 01EX514), Waltham, MA, USA, 19-22 August 2001; IEEE: Piscataway, NJ, USA, 2001; pp. 5-8.

5. Hu, B.; Beaulieu, N.C. Accurate evaluation of multiple-access performance in th-ppm and th-bpsk uwb systems. IEEE Trans. Commun. 2004, 52, 1758-1766. [CrossRef]

6. Khalesi, H.; Ghods, V. QPSK Modulation Scheme Based on Orthogonal Gaussian Pulses for IR-UWB Communication Systems. J. Circuits Syst. Comput. 2019, 28, 1950008. [CrossRef]

7. Sharma, S.; Gupta, A.; Bhatia, V. A simple modified peak detection based UWB receiver for WSN and IoT application. In Proceedings of the 2017 IEEE 85th Vehicular Technology Conference (VTC Spring), Sydney, NSW, Australia, 4-7 June 2017; IEEE: Piscataway, NJ, USA, 2017; pp. 1-6.

8. Benotmane, N.B.; Elahmar, S.A.; Dayoub, I.; Hamouda, W. Improved eigenfilter design method for channel shortening equalizer in TH-UWB. IEEE Trans. Veh. Technol. 2018, 67, 7749-7753. [CrossRef]

9. Zhao, L.; Haimovich, A.M. Capacity of m-ary ppm ultra-wideband communications over awgn channels. In Proceedings of the IEEE 54th Vehicular Technology Conference, VTC Fall 2001, Proceedings (Cat. No. 01CH37211), Atlantic City, NJ, USA, 7-11 October 2001; IEEE: Piscataway, NJ, USA, 2001; Volume 2, pp. 1191-1195.

10. Venkatesan, V.; Liu, H.; Nilsen, C.; Kyker, R.; Magana, M.E. Performance of an optimally spaced ppm ultra-wideband system with direct sequence spreading for multiple access'. In Proceedings of the 2003 IEEE 58th Vehicular Technology Conference, VTC 2003-Fall (IEEE Cat. No. 03CH37484), Orlando, FL, USA, 6-9 October 2003; IEEE: Piscataway, NJ, USA, 2003; Volume 1, pp. 602-606.

11. Wu, J.; Xiang, H.; Tian, Z. Weighted noncoherent receivers for uwb ppm signals. IEEE Commun. Lett. 2006, 10, 655-657.

12. Almodovar-Faria, J.M.; McNair, J. Optimal integration time for energy-detection ppm uwb systems. In Proceedings of the 2012 IEEE Global Communications Conference (GLOBECOM), Anaheim, CA, USA, 3-7 December 2012; IEEE: Piscataway, NJ, USA, 2012; pp. 4054-4059.

13. Yin, H.B.; Yang, J.A.; Wang, W.D. A randomly permuted fourier measurement matrix for uwb-ppm signals. Appl. Mech. Mater. 2014, 556, 2646-2649. [CrossRef]

14. Yajnanarayana, V.; Händel, P. Joint estimation of toa and ppm symbols using sub-nyquist sampled ir-uwb signal. IEEE Commun. Lett. 2017, 21, 949-952. [CrossRef]

15. Jabbar, M. Improving PSD of PPM-IR for UWB Signal Using Turbo Encoder. J. Ind. Eng. Manag. S 2018, 3, 2169-0316. [CrossRef] 
16. Goyal, V.; Dhaliwal, B. Improving ultra wideband (uwb) system by modified random combination of pulses. Eng. Rev. 2018, 38, 189-203. [CrossRef]

17. Maali, A.; Boukhelifa, A.; Mesloub, A.; Sadoudi, S.; Benssalah, M. An enhanced pulse position modulation (ppm) for both ir-uwb and dcc-uwb communication. In Proceedings of the 2019 13th European Conference on Antennas and Propagation (EuCAP), Krakow, Poland, 31 March-5 April 2019; IEEE: Piscataway, NJ, USA, 2019; pp. 1-5.

18. Daniels, D.J. Ground penetrating radar. In Encyclopedia of RF and Microwave Engineering; John Wiley \& Sons, Inc.: New York, NY, USA, 2005.

19. Cao, Y.; Labuz, J.; Guzina, B. Evaluation of pavement system based on ground-penetrating radar full-waveform simulation. Transp. Res. Rec. 2011, 2227, 71-78. [CrossRef]

20. Karim, H.H.; Al-Qaissi, A.M. Assessment of the accuracy of road flexible and rigid pavement layers using gpr. Eng. Technol. J. Part (A) Eng. 2014, 32, 788-799.

21. Nakayama, Y.; Kohno, R. Novel variable spreading sequence length system for improving the processing speed of ds-uwb radar. In Proceedings of the 2008 8th International Conference on ITS Telecommunications, Phuket, Thailand, 24 October 2008; IEEE: Piscataway, NJ, USA, 2008; pp. 357-361.

22. Dabove, P.; Di Pietra, V.; Piras, M.; Jabbar, A.A.; Kazim, S.A. Indoor positioning using Ultra-wide band (UWB) technologies: Positioning accuracies and sensors performances. In Proceedings of the 2018 IEEE/ION Position, Location and Navigation Symposium (PLANS), Monterey, CA, USA, 23-26 April 2018; IEEE: Piscataway, NJ, USA, 2018; pp. 175-184.

23. Xie, W.; Li, X.; Long, X. Underground operator monitoring platform based on ultra-wide band WSN. Int. J. Online Biomed. Eng. (iJOE) 2018, 14, 219-229. [CrossRef]

24. Huyen, N.T.; Hiep, P.T. Proposing adaptive PN sequence length scheme for testing non-destructive structure using DS-UWB'. In Proceedings of the 2019 3rd International Conference on Recent Advances in Signal Processing, Telecommunications \& Computing (SigTelCom), Hanoi, Vietnam, 21-22 March 2019; IEEE: Piscataway, NJ, USA, 2019; pp. 10-14.

25. Chen, X.; Kiaei, S. Monocycle shapes for ultra wideband system. In Proceedings of the 2002 IEEE International Symposium on Circuits and Systems, Proceedings (Cat. No. 02CH37353), Phoenix-Scottsdale, AZ, USA, 26-29 May 2002; IEEE: Piscataway, NJ, USA, 2002; Volume 1, pp. I-597-I-600.

26. De Abreu, G.T.F.; Mitchell, C.J.; Kohno, R. On the orthogonality of hermite pulses for ultra wideband communications systems. In Proceedings of the 6th International Symposium on Wireless Personal Multimedia Communications (WPMC), Yokosuka, Japan, 21-22 October 2003.

27. Gao, X. Uwb Indoor Localization System. Ph.D. Thesis, The George Washington University, Washington, DC, USA, 2018.

28. Kanzow, C.; Yamashita, N.; Fukushima, M. Withdrawn: Levenberg-marquardt methods with strong local convergence properties for solving nonlinear equations with convex constraints. J. Comput. Appl. Math. 2005, 173, 321-343. [CrossRef]

29. Benedetto, D. Understanding ultra wide band radio fundamentals. In Understanding Ultra Wide Band Radio Fundamentals; Pearson Education India: Delhi, India, 2008.

30. Hubbard, S.S.; Peterson, J.E., Jr.; Majer, E.L.; Zawislanski, P.T.; Williams, K.H.; Roberts, J.; Wobber, F. Estimation of permeable pathways and water content using tomographic radar data. Lead. Edge J. 1997, 16, 1623-1630. [CrossRef]

(c) 2020 by the authors. Licensee MDPI, Basel, Switzerland. This article is an open access article distributed under the terms and conditions of the Creative Commons Attribution (CC BY) license (http:/ / creativecommons.org/licenses/by/4.0/). 